\title{
Risk Scores for Stroke Prevention in Patients with Non-Valvular Atrial Prevention: Is it Time to Put Parameters of Left Atrial Appendage Morphology and Function Into Clinical Practice?
}

\author{
Angelo A. V. de Paola \\ Escola Paulista de Medicina - UNIFESP, São Paulo, SP - Brazil \\ Editorial related to the article: Association between Morphodynamic Variables by Transesophageal Echocardiography and CHA $\mathrm{DS}_{2}-V_{\text {asc }}$ Values
}

Overall, one-third of thromboembolic events originated from the heart. Left atrial appendage (LAA) and its anatomic complexity has been implicated in $90 \%$ of cases of thrombus formation and cardioembolic events in patients with non-valvular atrial fibrillation (AF). Risk scores to predict thromboembolism from AF, such as $\mathrm{CHADS}_{2}$ and $\mathrm{CHA}_{2} \mathrm{DS}_{2}$ VASc are widely used to guide anticoagulation therapy ${ }^{1}$ and varies in relation to comorbidities associated with the thrombogenic substrate; however, because of its clinical conception, they did not include atrial anatomy (atrial enlargement), function (flow velocities) and electrophysiology (atrial fibrillation burden). Patients with $\mathrm{CHA}_{2} \mathrm{DS}_{2}$ VASc score $\geq 2$ are at intermediate or higher risk (event rate $\geq$ $2-3 \% /$ year) and anticoagulation is recommended. Left atrial variables such as LAA function and morphology, routinely studied by transesophageal echocardiography (TEE), were not considered in these scores.

In 2012, Di Biase et al.; published a retrospective study of 932 patients with AF and computed tomography (CT)/magnetic resonance imaging (MRI) classification of four different types of LAA morphologies: chicken wing, cactus, windsock and cauliflower. Non-chicken wing categories were 4 to 8 times more likely to have had stroke/TIA. Eight additional cross-sectional studies utilizing this classification for LAA morphology in 2,210 patients ( 6 with CT and 2 with TEE), found a significant

\section{Keywords}

Stroke; Atrial Fibrillation; Atrial Apendage; Arrhytmias, Cardiacs; Myocardial Contraction; Echocardiography, Transesophageal. association with non-chicken-wing morphology and stroke in 5 series $^{3-7}$ and no significant difference in 3 other studies. ${ }^{8-10}$

In this issue of Int J Cardiovasc Sci, Linhares et al., ${ }^{11}$ readdress the question of LAA morphology and stroke risk studying 237 patients with transesophageal echocardiography (TEE), the gold standard and most clinical utilized tool for thrombus detection in patients with LAA thrombosis/stroke risk. In this paper, the possibility of simple characterization with TEE patients with less-thrombogenic LAA anatomy (smooth internal surface and sharp angulation - "the chicken wing morphology"), was associated with less significant clinical events compared with patients with "thrombogenic non-chicken LAA morphology" (11.2\% vs. $25.2 \%-p=0.005)$.

Because of the proximity of the esophagus to the left atrium, high-frequency transducers are able to acquire images of LA, especially LAA, using TEE, the test of choice for scanning these structures, with the possibility of detecting LAA trabeculations, pectinate muscles, number of LAA lobes, LAA velocities and thrombus. Additionally, its safety and semi-invasiveness make it the most sensitive and clinically useful diagnostic technique used for searching cardiac embolic sources, representing the mainstay of investigation in patients suspected to have cerebral embolism.

LAA is the only part from the primitive left atrium (LA) and has pectinate muscle and trabeculations. Smoothness of LAA, prevalence of single lobes and acute angle bend ("chicken wing") are the morphologic variables of LAA described as non-thrombogenic and may be reproducibly recognized by TEE. On the other 
hand, the appendage flow velocity can be obtained with pulsed Doppler interrogation and $<55 \mathrm{~cm} / \mathrm{s}$ velocity appears to be related to thrombus formation. In these conditions, slow-swirling echoes resembling smoky haze and "spontaneous echo-contrast" (SEC) are findings that physiologically represent the conditions for LAA thrombogenesis. Flow velocities in patients with "chicken-wing LAA" are higher than other LAA morphologies, potentially reducing stasis, thrombus formation and stroke risk. It is possible that the "acute angle" in these cases may be protective against stroke by providing a milieu in which thrombi are less likely to form.

TEE is widely utilized in patients prior to cardioversion or AF ablation to rule out the presence of LAA thrombus, providing images with important anatomic and functional information that has not been incorporated into clinical risk scores. This additional information regarding LAA morphology and function may be important in situations where AF is "eliminated", helping to decide over controversies such as withheld anticoagulation therapy in selected patients after successful catheter ablation and no recurrences of AF.

This simple echocardiographic classification of LAA anatomy may help to identify patients with cardioembolic risk of stroke subtypes in patients with AF and clinical conditions, where TEE was indicated. Larger studies are needed to validate these findings. Patients with "malignant LAA morphologies" deserve special attention especially for difficult and critical clinical decisions in the anticoagulation scenario for stroke prevention of patients with AF.

It is time to have a different approach for patients where the risk/benefit decision for anticoagulation is provocative, such as post-ablation AF patients. Designing studies with risk scores including LA/LAA morphology and function may provide important information and, hopefully, allow to put these parameters into clinical practice!

\section{References}

1. Kirchhof P, Benussi S, Kotecha D, Ahlsson A, Atar D, Casadei B, et al; 2016 ESC Guidelines for the management of atrial fibrillation developed in collaboration with EACTS. Eur Heart J. 2016;37(38)2893-962.

2. Di Biase L, Santangeli P, Anselmino M, Mohanty P, Salvetti I, Gili S, et al . Does the left atrial appendage morphology correlate with the risk of stroke in patients with atrial fibrillation? Results from a multicenter study. J Am Coll Cardiol. 2012; 60(6):531-8.

3. Lee JM, Seo J, Uhm JS, Kim YJ, Lee HJ, Kim JY, et al. Why is left atrial appendage morphology related to strokes? An analysis of the flow velocity and orifice size of theleft atrial appendage. J Cardiovasc Electrophysiol. 2015;26(9):922-7

4. Lee Y, Park HC, Lee Y, Kim SG. Comparison of morphologic features and flow velocity of the left atrial appendage among patients with atrial fibrillation alone, transient ischemic attack, and cardioembolic stroke. Am J Cardiol. 2017;119(10):1596-604

5. Kimura T, Takatsuki S, Inagawa $K$, Katsumata $Y$, Nishiyama $T$, Nishiyama N, et al. Anatomical characteristics of the left atrial appendage in cardiogenic stroke with low chads2 scores. Heart Rhythm. 2013;10(6):921-5

6. Anselmino M, Scaglione M, Di Biase L, Gili S, Santangeli P, Corsinovi $\mathrm{L}$, et al. Left atrial appendage morphology and silent cerebral ischemia in patients with atrial fibrillation. Heart Rhythm. 2014;11(1):2-7.

7. Korhonen M, Muuronen A, Arponen O, Mustonen P, Hedman M, Jakala $\mathrm{P}$, et al. Left atrial appendage morphology in patients with suspected cardiogenic stroke without known atrial fibrillation. PloS One. 2015;10(3):e0118822.

8. Nedios S, Kornej J, Koutalas E, Bertagnolli L, Kosiuk J, Rolf S, et al. Left atrial appendage morphology and thromboembolic risk after catheter ablation for atrial fibrillation. Heart Rhythm. 2014;11(12):2239-46.

9. Petersen M, Roehrich A, Balzer J, Shin DI, Meyer C, Kelm M, et al. Left atrial appendage morphology is closely associated with specific echocardiographic flow pattern in patients with atrial fibrillation. Europace . 2015;17(4):539-45.

10. Khurram IM, Dewire J, Mager M, Maqbool F, Zimmerman SL, Zipunnikov V, et al. Relationship between left atrial appendage morphology and stroke in patients with atrial fibrillation. Heart Rhythm. 2013;10(12):1843-9.

11. Linhares RJ, Moreira DA, Peixoto LB, Da Cruz AP, Barretto RB, Le Bihan DC,et al. Association between morphodynamic variables by transesophageal echocardiography and CHA2DS2-Vasc values. Int J Cardiovasc Sci. 2019;32(5):460-470. 